\title{
Dynamic Interaction of the "Bridge-Train" System on High-Speed Railways
}

\author{
Leonid Diachenko ${ }^{1,}$, , Vladimir Smirnov ${ }^{2}$ \\ ${ }^{1,2}$ Emperor Alexander I St. Petersburg State Transport University, Moskovsky ave., 9, Saint- \\ Petersburg, 190031, Russia
}

\begin{abstract}
This work contains the results of a research of the dynamic processes in the "bridge-train" system while passenger trains move over a bridge structure (overpass) in high-speed. The article presents the methodology of mathematic modelling, and the basic differential equations of the studied system elements motion are provided. Also there is a description of dynamic interaction of the bridge-train system numerical model based on the FEM. In general, taking into account in the design scheme of the "bridge" system not only spans, but also piers with a foundation, it is possible to more accurately determine the values of the bridge natural frequencies, which is a key factor in assessing the dynamic response of a structure when passing a high-speed train.
\end{abstract}

\section{Introduction}

According to research [1-5], in conditions of high-speed train traffic, the inertial forces in the bridge structure and the railway track elements, that occur under the impact of the rolling stock load which usually consists of the same type of cars, increase. These effects are particularly important for bridge structures of the trestle type with a large number of the same length girder spans and high height supports.

Thus, the study of dynamic processes in train cars, span structures and piers of bridges becomes relevant. Obviously, that the estimated model of the system "bridge-train" should include: high-speed train model (including two-stage spring suspension), track structure model (including elastic and inertial characteristics), superstructure and the nature of its support (including support friction in moving parts), supports and foundations (including the ductility of the base).

A significant role on high-speed railway bridges is also played by "irregularities" of track, which might be periodic, due to the value of the construction lift and span structures elastic deflections, deformed under the live load, or accidental, characterized by defects in the rails and wheels of the train.

Track irregularities become a source of oscillations "bridge-train" system along with the main effect in the form of periodic passage of bogies, or wheelsets, rolling stock along spans. As is known [6-7], the bridge dynamic response magnitude under the influence of a high-speed train is determined primarily by the parameters of the system itself (car lengths,

* Corresponding author: leonid_dyachenko@mail.ru 
axial loads, bridge static structure, span lengths, structural material, stiffness and massiveness of beams and supports, etc.). The dynamic response of train cars is also determined by the rolling stock parameters (sprung and unsprung masses, stiffness and damping in suspension, etc.) and the bridge (length of beams and their number, construction scheme, track parameters on the bridge, etc.).

\section{Problem statement}

When deriving a system of differential equations describing the movement of "bridge-train" system elements during their interaction, one should take into account the high complexity, as a result of which it seems appropriate to maximize the idealization of the calculation model, providing for a number of assumptions:

- the bridge is accepted as a regular system (equal spans);

- the abutments of the bridge are absolutely rigid;

- oscillations of the bridge and train elements are investigated in a 2D statement;

- the train is modeled as a sequential chain of the same type of biaxial cars.

After these simplifications, the design scheme of the "bridge-train" dynamic interaction system will have the form shown in Fig. 1.

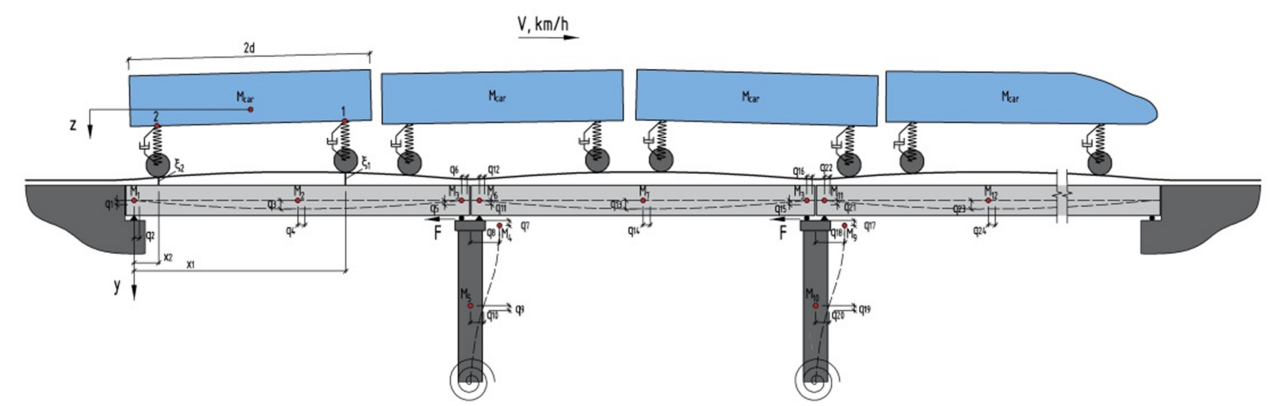

Fig. 1. Model for «bridge-train» dynamic interaction analysis

The assumptions made allow us to write down the differential equations of oscillations of the "bridge-train" system elements of in the following form:

$$
\begin{gathered}
q_{1}=0 \\
q_{2}=0 \\
q_{3}=-\left(M_{2} \ddot{q}_{3}+\beta \dot{q}_{3}\right)\left(\delta_{33}+\delta_{55} / 2\right)-M_{3} \ddot{q}_{5} \delta_{35}-M_{4} \ddot{q}_{7} \delta_{37}-M_{5} \ddot{q}_{10} \delta_{3,10} \\
-\sum_{i=1}^{k} \frac{M_{\ni}}{2}\left(\ddot{z}_{1}-q\right) \delta_{3,1, i}-\sum_{i=1}^{l} \frac{M_{\ni}}{2}\left(\ddot{z}_{2}-q\right) \delta_{3,2, i}+\zeta F_{1} \operatorname{sign}\left(\dot{q}_{3}+\dot{q}_{6}-\dot{q}_{12}\right) \cdot \ldots \\
q_{7}=-\left(M_{4} \ddot{q}_{7}+\beta \dot{q}_{7}\right) \delta_{77}-M_{3} \ddot{q}_{5} \delta_{57}-M_{6} \ddot{q}_{11} \delta_{11,7}-\sum_{i=1}^{k} \frac{M_{\ni}}{2}\left(\ddot{z}_{1}-q\right) \delta_{7,1, i}- \\
-\sum_{i=1}^{l} \frac{M_{\ni}}{2}\left(\ddot{z}_{1}-q\right) \delta_{7,2, i}-\sum_{i=1}^{m} \frac{M_{\ni}}{2}\left(\ddot{z}_{1}-q\right) \delta_{7,1, i}-\sum_{i=1}^{n} \frac{M_{\ni}}{2}\left(\ddot{z}_{1}-q\right) \delta_{7,2, i} \\
q_{8}=-\left(M_{4} \ddot{q}_{8}+\beta \dot{q}_{8}\right) \delta_{88}+F_{1} \operatorname{sign}\left(\dot{q}_{6}-\dot{q}_{12}\right) \delta_{88}-\sum_{i=1}^{k} \frac{M_{\ni}}{2}\left(\ddot{z}_{1}-q\right) \delta_{8,1, i}- \\
\sum_{i=1}^{l} \frac{M_{\ni}}{2}\left(\ddot{z}_{2}-q\right) \delta_{8,2, i}-\sum_{i=1}^{m} \frac{M_{\ni}}{2}\left(\ddot{z}_{1}-q\right) \delta_{8,1, i}-\sum_{i=1}^{n} \frac{M_{\ni}}{2}\left(\ddot{z}_{2}-q\right) \delta_{8,2, i}+ \\
+F_{1} \operatorname{sign}\left(\dot{q}_{6}-\dot{q}_{12}\right) \cdot \ldots
\end{gathered}
$$




$$
\begin{gathered}
q_{10}=-\left(M_{5} \ddot{q}_{10}+\beta \dot{q}_{10}\right) \delta_{10,10}+F_{1} \operatorname{sign}\left(\dot{q}_{6}-\dot{q}_{12}\right) \delta_{10,8} \\
\ldots \\
q_{13}=-\left(M_{7} \ddot{q}_{13}+\beta \dot{q}_{13}\right)\left(\delta_{13,13}+\delta_{11} / 2+\delta_{15} / 2\right)-M_{5} \ddot{q}_{11} \delta_{11,13}-M_{8} \ddot{q}_{15} \delta_{15,13}- \\
M_{4} \ddot{q}_{7} \delta_{7,13}-M_{9} \ddot{q}_{9} \delta_{7,18}-\sum_{i=1}^{k} \frac{M_{\ni}}{2}\left(\ddot{z}_{1}-q\right) \delta_{13,1, i}-\sum_{i=1}^{l} \frac{M_{\ni}}{2}\left(\ddot{z}_{2}-q\right) \delta_{13,2, i}- \\
\sum_{i=1}^{m} \frac{M_{\ni}}{2}\left(\ddot{z}_{1}-q\right) \delta_{13,1, i}-\sum_{i=1}^{n} \frac{M_{\ni}}{2}\left(\ddot{z}_{2}-q\right) \delta_{13,2, i}+\zeta F_{1} \operatorname{sign}\left(\dot{q}_{13}+\dot{q}_{16}-\dot{q}_{22}\right) \cdot \ldots
\end{gathered}
$$

Here $z_{1}$ - the absolute movement of the body of the i-th car at point 1 (the first in the direction of travel), determined by the stiffness of the springs and track irregularities;

$z_{2}$ - the same at point 2 ;

$q_{j}$ - movement of the bridge point $\mathrm{j}$ in the direction of the generalized coordinate;

$\delta_{k, l}$ - unit displacement of bridge point $\mathrm{k}$ from the force applied at point $\mathrm{l}$;

$M_{\text {э }}$ - sprung mass of the car;

$M_{i}$ - i-th discrete mass of the bridge;

$F_{i}$ - friction force in movable bearings mounted on the i-th support from permanent and live loads;

$\beta$ - damping coefficient of the bridge element;

$g$ - acceleration of gravity force;

$\zeta$ - the coefficient of transition from vertical displacements of the middle of the span to longitudinal displacements of the same point in the level of the hinge of the supporting part.

Trains car oscillations are largely determined by the deformation of the springs and the track irregularities. You can write:

$$
\begin{aligned}
& \Delta_{1}=z_{1}-y_{1}^{\ni}+\xi_{1} \\
& \Delta_{2}=z_{2}-y_{2}^{\ni}+\xi_{2}
\end{aligned}
$$

, where $\Delta_{1}, \Delta_{2}$ - spring deformations, respectively, under point 1 and 2 of the i-th car;

$y_{1}^{\ni}, y_{2}^{\ni}$ - deflection of the bridge beam at points 1 and 2, respectively, of the i-th car;

$\xi_{1}, \xi_{2}$ - ordinates of "irregularities" of the way at points 1 and 2 of the i-th car.

At the same time, inertia forces $X_{1}, X_{2}$ arise at points 1 and 2 of the car, determined from the equations:

$$
\begin{gathered}
X_{1}+X_{2}=\frac{M_{\ni}}{2} \ddot{z}_{1}+\frac{M_{\ni}}{2} \ddot{z}_{2}, \\
\left(X_{1}-X_{2}\right) 2 d=\frac{J \ddot{z}_{1}-J \ddot{z}_{2}}{2 d}, \\
X_{1}=\frac{M_{\ni}}{4}\left(\ddot{z}_{1}+\ddot{z}_{2}\right)+\frac{J}{8 d^{2}}\left(\ddot{z}_{1}-\ddot{z}_{2}\right)
\end{gathered}
$$




$$
X_{2}=\frac{M_{\ni}}{4}\left(\ddot{z}_{1}+\ddot{z}_{2}\right)-\frac{J}{8 d^{2}}\left(\ddot{z}_{1}-\ddot{z}_{2}\right)
$$

Here $J_{-}$is the moment of inertia of the sprung part of the car relative to the central main axis of inertia, determined by the formula:

$$
J=M_{\ni} r^{2}
$$

where $r$ - is the radius of inertia of the car;

Expressions for unknowns $z_{1}, z_{2}$, you can apply the following:

$$
\begin{aligned}
& z_{1}=\Delta_{1}+y_{1}^{\ni}-\xi_{1} \\
& z_{2}=\Delta_{2}+y_{2}^{\ni}-\xi_{2}
\end{aligned}
$$

From these expressions, the values $\ddot{z}_{1}, \ddot{z}_{2}$ are found to obtain the displacements of the bridge points in the directions of the generalized coordinate $q_{j}$ and inertia forces $X_{1}, X_{2}$ of points 1 and 2 of the car.

You can apply:

$$
\Delta_{1}=\Delta_{2}=\frac{M_{3}}{2 \delta}
$$

where $\delta$ - is the compliance of the spring suspension of the car;

Deflections of the beam of the span under the first and second axes of the i-th car are determined by the expressions:

$$
\begin{aligned}
& y_{1}^{\ni}=y_{3} \sin \frac{\pi x_{1}}{l}+\frac{y_{5}}{2} \cdot \frac{x_{1}}{l} \\
& y_{2}^{\ni}=y_{3} \sin \frac{\pi x_{2}}{l}+y_{5}
\end{aligned}
$$

Denote $\frac{x_{1}}{l}=U_{1}, \frac{x_{2}}{l}=U_{2}$, then for the first span you can write:

$$
\begin{aligned}
& \delta_{3,1 \ni}=\delta_{33} \sin \pi U_{1}+\frac{\delta_{55}}{2} \cdot U_{1} \\
& \delta_{3,2 \ni}=\delta_{33} \sin \pi U_{2}+\frac{\delta_{55}}{2} \cdot U_{2}
\end{aligned}
$$

Given that $x=V t$, where ( $V-$ is the speed of the train, and $t_{-}$is the time), we can represent the coefficients of influence in the form:

$$
\begin{aligned}
& \delta_{3,1 \ni}=\delta_{33} \sin \pi \frac{V t_{1}}{l}+\frac{\delta_{55}}{2} \cdot \frac{V t_{1}}{l} \\
& \delta_{3,2 \ni}=\delta_{33} \sin \pi \frac{V t_{2}}{l}+\frac{\delta_{55}}{2} \cdot \frac{V t_{2}}{l}
\end{aligned}
$$

And finally get the dependence of the "bridge-train" system dynamic characteristics on the speed of the train. The presented model allows us to evaluate the nature of the "bridgetrain" interaction, however, it is based on rather crude simplifications. For more accurate 
practical calculations, a numerical model of the "bridge-train" system interaction based on the FEM can be used [9].

\section{Analysis of train-bridge dynamic interaction}

When performing the calculations, the following parameters of the bridge structure and rolling stock were adopted:

- Bridge scheme: 10x34.2 m;

- Spans: box-section of prestressed concrete $\left(\mathrm{E}=36000 \mathrm{MPa}, \mathrm{A}=9.58 \mathrm{~m}^{2}\right.$, $\mathrm{J}=11.68 \mathrm{~m}^{4}$ ). The mass of ballastless track was included in the mass of spans;

- Intermediate supports: continuous section of ordinary reinforced concrete $\left(\mathrm{E}=36000 \mathrm{MPa}, \mathrm{A}=11.19 \mathrm{~m}^{2}, \mathrm{~J}=23.66 \mathrm{~m}^{4}\right)$ with a height of 10 to $25 \mathrm{~m}$;

- Foundation of piers: bored piles $\varnothing 1.5 \mathrm{~m}$, length $25.0 \mathrm{~m}$ [8];

- Rolling stock: high-speed train "Sapsan" (Velaro Rus), car length 24.73 m, axle load $147 \ldots 165 \mathrm{kN}$, boogie base $2.6 \mathrm{~m}$, number of cars 10 .

At the first stage of the calculation, a modal analysis of the "bridge-track" system was performed. Some general forms and corresponding eigenfrequencies are shown in Fig 2.
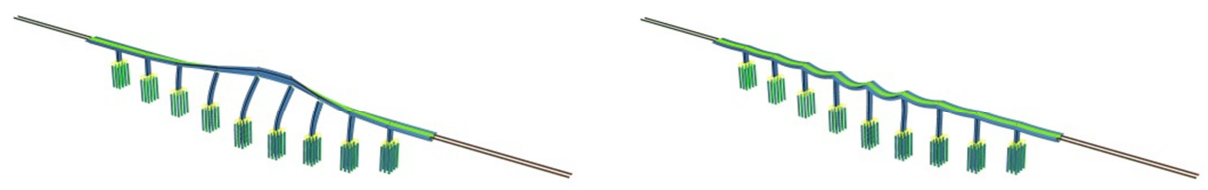

a).

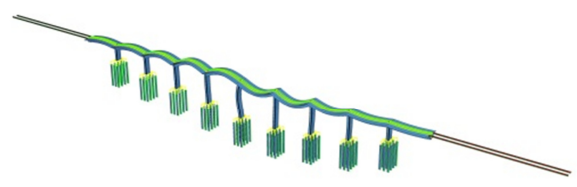

b).

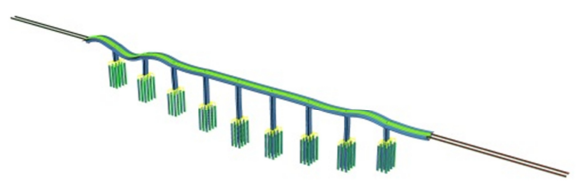

c).

d).

Fig. 2. Natural frequencies of "bridge-track" system. a). $1^{\text {st }}-1.25 \mathrm{~Hz}$; b). $9^{\text {th }}-4.35 \mathrm{~Hz} ;$ c). $10^{\text {th }}-4.40$ $\mathrm{Hz}$; d). $17^{\text {th }}-4.90 \mathrm{~Hz}$

To evaluation the nature of the "bridge-train" system dynamic vibrations, the calculated case of the movement of a high-speed train at a speed of $387.3 \mathrm{~km} / \mathrm{h}$ was considered, which for a span of $34.2 \mathrm{~m}$ (the first natural frequency of vertical bending vibrations $-4.35 \mathrm{~Hz}$ ) is a critical (resonant) speed.

In the course of the calculations, vertical displacements (Fig.3), accelerations (Fig.4) of car bodies and the middle of the bridge spans (Fig.5, 6), as well as longitudinal horizontal displacements of the piers (Fig.7) were obtained. 


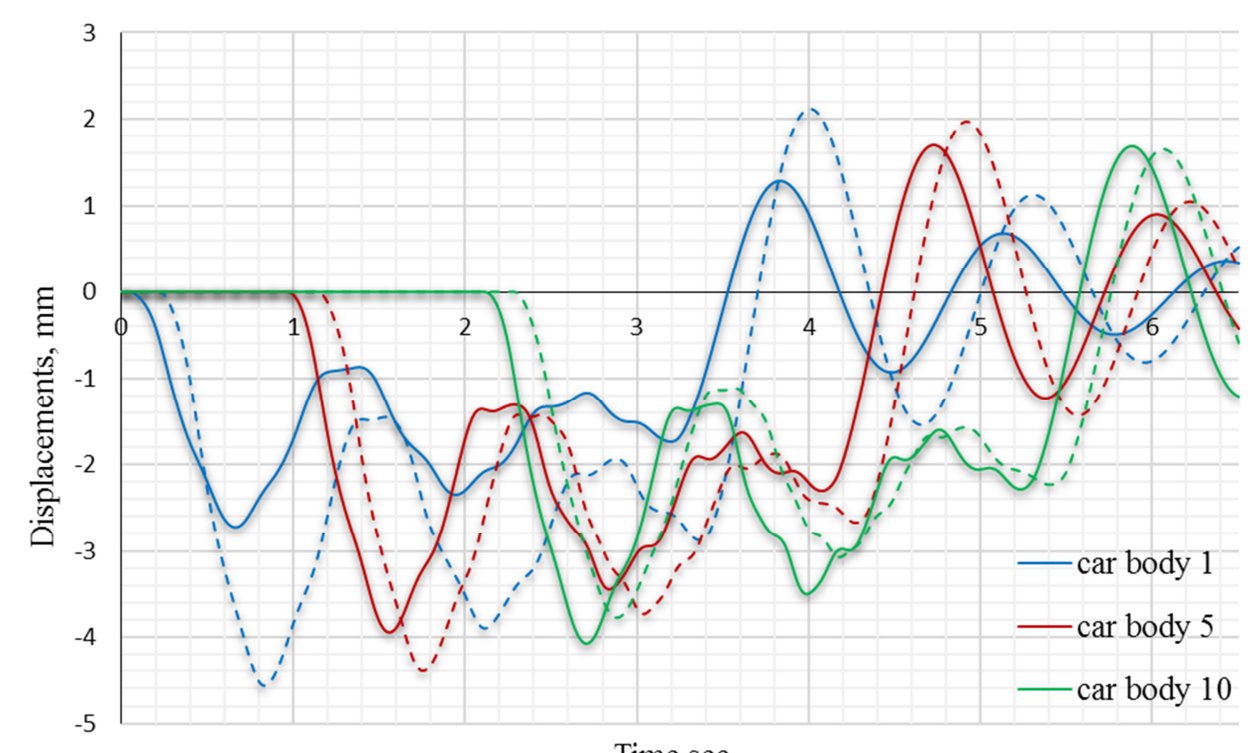

Time,sec

Fig. 3. Vertical displacements in the front (solid) and back (dashed) point of car bodies while moving of a high-speed train "Sapsan" (Velaro RUS) at a resonant speed of $387.3 \mathrm{~km} / \mathrm{h}$

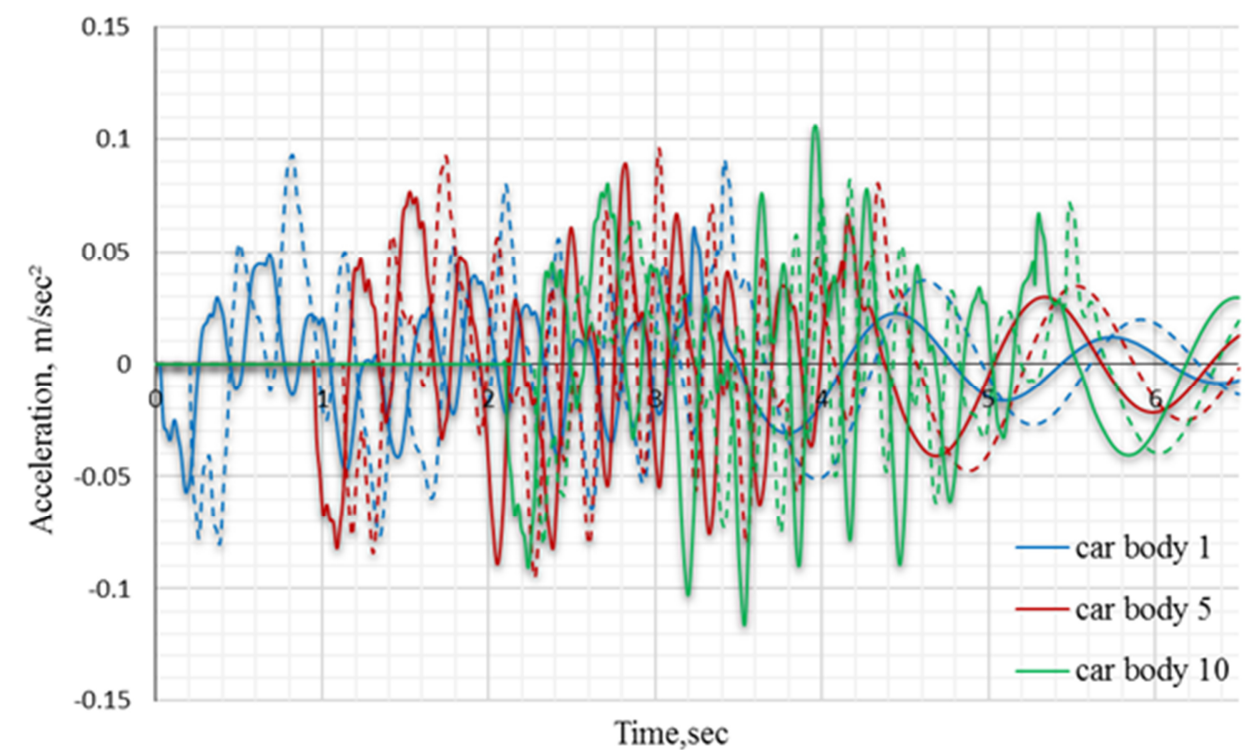

Fig. 4. Vertical accelerations in the front (solid) and back (dashed) point of car bodies while moving of a high-speed train "Sapsan" (Velaro RUS) at a resonant speed of $387.3 \mathrm{~km} / \mathrm{h}$ 


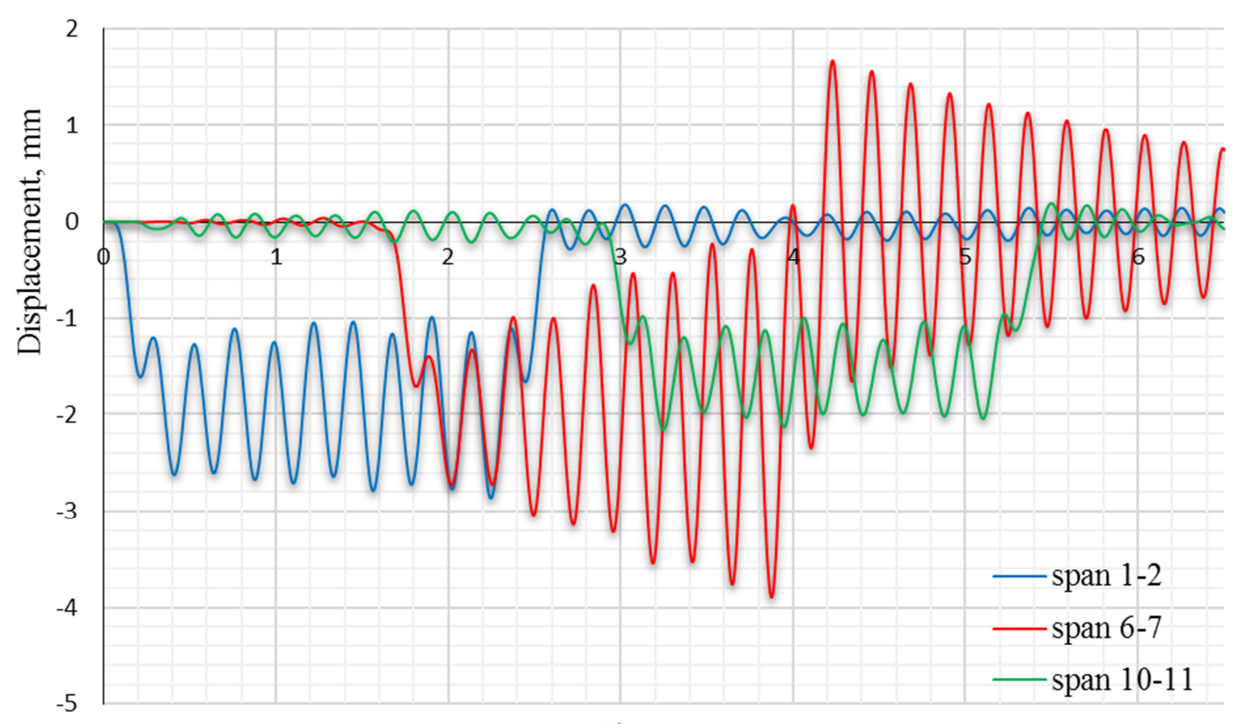

Time,sec

Fig. 5. Vertical displacements in the middle of the bridge spans (1-2, 6-7 and 10-11) while moving of a high-speed train "Sapsan" (Velaro RUS) at a resonant speed of $387.3 \mathrm{~km} / \mathrm{h}$

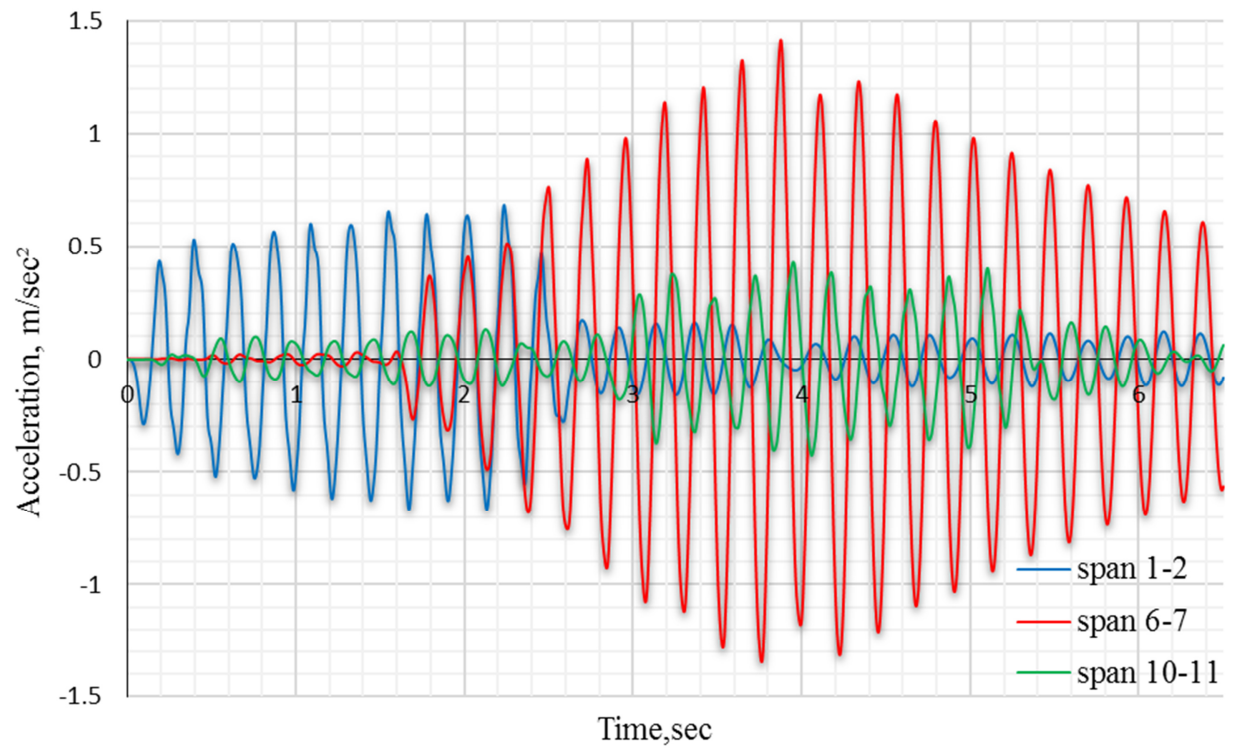

Fig. 6. Vertical accelerations in the middle of the bridge spans (1-2, 6-7 and 10-11) while moving of a high-speed train "Sapsan" (Velaro RUS) at a resonant speed of $387.3 \mathrm{~km} / \mathrm{h}$ 


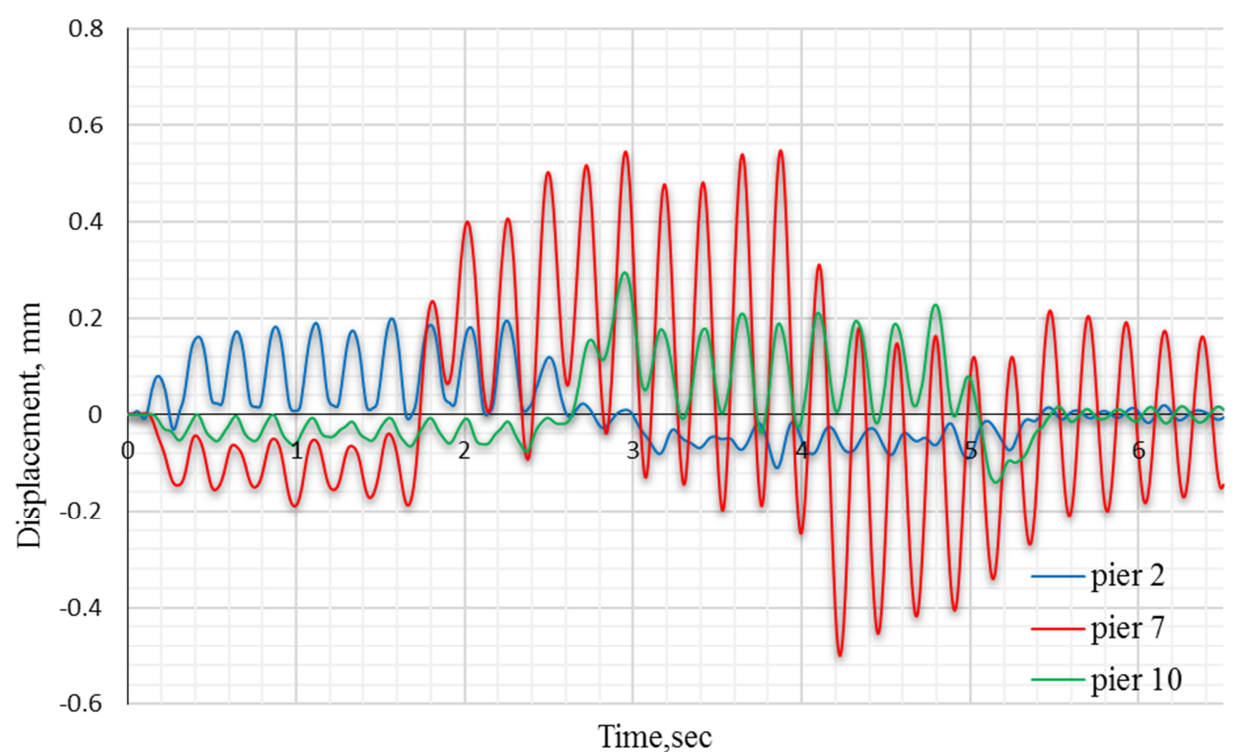

Fig. 7. Longitudinal horizontal displacements of the bridge piers $\left(2^{\text {nd }}, 7^{\text {th }}\right.$ and $\left.10^{\text {th }}\right)$ while moving of a high-speed train "Sapsan" (Velaro RUS) at a resonant speed of $387.3 \mathrm{~km} / \mathrm{h}$.

\section{Discussion}

As a result of the calculations, the values of the bridge spans natural frequencies were obtained, which have values of $4.35-4.90 \mathrm{~Hz}$. The difference in the value of the natural frequency for different spans is explained by the inclusion in the coupled vibrations of piers with different height. For spans on high piers, the natural frequency value is lower. Also, this factor determines various values of train speeds at which resonant span vibrations will occur.

It should be noted that the interaction of the bridge piers, spans and jointless track leads to a decrease in the amplitudes of vibrations compared to the usual simplified model [6-7] by about $30-40 \%$. However, this fact has a negative impact on the dynamic work of the system. At close values of the spans and piers eigenfrequencies, vertical resonance vibrations are transmitted to the piers and cause intense longitudinal vibrations of the piers, which necessitates taking into account dynamic effects in pier designing (especially high) bridges on high-speed highways.

\section{Conclusion}

Analysis of the studies performed allows us to conclude that when a high-speed train passes through a regular type bridge, all elements of the "bridge-train" system interact actively. Under certain conditions, the values of the natural bending natural frequencies of the spans and piers can be close, which will lead to the transfer of additional horizontal forces to the piers during intense resonant vibrations of the spans.

In general, taking into account in the design scheme of the "bridge" system not only spans, but also piers with a foundation, it is possible to more accurately determine the values of the bridge natural frequencies, which is a key factor in assessing the dynamic response of a structure when passing a high-speed train. 
It should also be noted that the introduction of the parameters of the soil foundation into the design scheme has a beneficial effect on the operation of the entire system, since part of the spans vibrations energy is transmitted through the supports and the foundation to the ground and dissipated, increasing the dissipative characteristics of the entire system.

\section{References}

1. S.G.M. Neves, A. Azevedo, Rui Calçada, IABMAS 2008 - 4th International Conference on Bridge Maintenance, Safety and Management (2008). DOI: 10.1201/9781439828434.ch79

2. R. Delgado, SM. Santos. Computers \& Structures 63, 511-523 (1997). DOI:10.1016/S0045-7949(96)00360-4

3. N. Zhang, H. Xia, W. Guo, Journal of Sound and Vibration 309(3-5), 407-425 (2008)

4. Y. B.Yang, J. D. Yau, Y. S. Wu, Vehicle-Bridge Interaction Dynamics: With Applications To High-Speed Railways (Singapore, World Scientific Publishing Company, 2004)

5. S.G.M. Neves, P.A. Montenegro, A.F.M. Azevedo, Rui Calçada, The Second International Conference on Railway Technology: Research, Development and Maintenance (2014). DOI: 10.4203/ccp.104.81

6. L. Dyachenko, A. Benin, A. Dyachenko, IOP Conf. Series: Materials Science and Engineering 463, 022048 (2018). DOI: 10.1088/1757-899X/463/2/022048

7. L. Diachenko, A. Benin, V. Smirnov, A. Diachenko, Civil and Environmental Engineering 14(1), 37-43 (2018). DOI: 10.2478/cee-2018-0005

8. V.N. Smirnov, L.K Dyachenko, A.O. Dyachenko, L.A. Andreeva, Procedia Engineering 189, 610-615 (2017). DOI: 10.1016/j.proeng.2017.05.097

9. L. Dyachenko, A. Benin, A. Dyachenko, MATEC Web of Conferences Siberian Transport Forum - TransSiberia (2018). DOI: 10.1051/matecconf/2018239050 